\title{
A NEW GRONWALL-BELLMAN TYPE INTEGRAL INEQUALITY AND ITS APPLICATION TO FRACTIONAL STOCHASTIC DIFFERENTIAL EQUATION
}

\author{
SOBIA RAFEEQ ${ }^{1}$ AND SABIR HUSSAIN ${ }^{2}$ \\ ${ }^{1,2}$ Department of Mathematics \\ University of Engineering and Technology \\ Lahore, PAKISTAN
}

\begin{abstract}
A Gronwall-Bellman type fractional integral inequality has been derived which is a generalization of already existing result. We also discussed the certain characteristics of the solution of a stochastic differential equation with the help of derived result.
\end{abstract}

AMS Subject Classification: 39B72, 26D10, 34A34

Key Words: integral inequality, fractional differential equation, qualitative properties

Received: November 27, 2018; Revised: February 22, 2019 ;

Published (online): February 25, $2019 \quad$ doi: $\quad 10.12732 /$ dsa.v28i2.3

Dynamic Publishers, Inc., Acad. Publishers, Ltd. https://acadsol.eu/dsa

\section{INTRODUCTION}

It is well known that inequalities play a significant role in the study of the qualitative behavior of solutions of differential, integral and integro-differential equations. Among others Gronwall-Bellman integral inequality plays a significant role to discuss the boundedness, global existence, uniqueness, stability, and continuous dependence of solutions to some certain differential equations, fractional differential equations, stochastic differential equations. Such inequalities have gained much attention of many researchers $[12,6,3,1,8,7,10,2,9,11,5]$. Recent paper is a motivation of an idea given by Q-X Kong et al. [4].

Moreover, our result can be used to analyze the behavior of solution of fractional stochastic differential equation. The paper is arranged in such a way that after this Introduction in Section 2, we give our main result and related consequences. In Section 3, we discuss the existence and uniqueness of the solution of a stochastic 
differential equation.

\section{MAIN RESULTS}

Lemma 1. [4] Let $a_{1}, a_{2} \in R$. Then for $\xi>0$, we have

$$
\frac{\Gamma\left(\xi+a_{1}\right)}{\Gamma\left(\xi+a_{2}\right)}=O\left(\xi^{a_{1}-a_{2}}\right), \quad \xi \rightarrow \infty .
$$

Definition 2. [4] Let $a_{2}>a_{1}>0, \varrho>0$. Then the following definition:

$$
F_{\varrho, a_{1}, a_{2}}(\xi):=\sum_{n=0}^{\infty} b_{n} \xi^{n}, \quad \xi \in \mathbf{R}
$$

is well defined, where $b_{0}$ is a positive constant, and $b_{n+1}=\left(\frac{\Gamma\left(n \varrho+a_{1}\right)}{\Gamma\left(n \varrho+a_{2}\right)}\right) b_{n}$.

Theorem 3. Let $g_{1}(\mathfrak{t})$ be a non-negative and locally integrable function on $\mathbf{R}^{+}$; let $g_{2}(\mathfrak{t}), g_{3}(\mathfrak{t})$ are nonnegative, nondecreasing continuous functions defined on $\mathbf{R}^{+}$and bounded. Further, if $r(\mathfrak{t})$ is a nonnegative and $\mathfrak{t}^{a-1} r(\mathfrak{t})$ is locally integrable on $\mathbf{R}^{+}$ such that:

$$
r(\mathfrak{t}) \leq g_{1}(\mathfrak{t})+g_{2}(\mathfrak{t}) \int_{0}^{\mathfrak{t}}(\mathfrak{t}-p)^{b-1} p^{a-1} r(p) d p+g_{3}(\mathfrak{t}) \int_{0}^{\mathfrak{t}} \mathfrak{t}^{b-1} p^{a-1} r(p) d p,
$$

for $\mathfrak{t} \in \mathbf{R}^{+}$. Then, for each constant $a>0,0<b<1, c=a+b-1>0, \omega>0$, $\mathfrak{t} \in[0, \omega], \theta, \eta \in N$, we have

$$
r(\mathfrak{t}) \leq \begin{cases}g_{1}(\mathfrak{t})+\sum_{\theta=1}^{\infty}(\Gamma(b))^{\theta-1} \prod_{i=1}^{\theta-1} \frac{\Gamma(i c)}{\Gamma(i c+b)} & \\ \times \sum_{\eta=0}^{\theta} C_{\eta}^{\theta} g_{2}^{\theta-\eta}(\mathfrak{t}) g_{3}^{\eta}(\mathfrak{t}) & a, b \in(0,1), \\ \times \int_{0}^{\mathfrak{t}}(\mathfrak{t}-p)^{\theta c-a} p^{a-1} g_{1}(p) d p, & \\ & \\ g_{1}(\mathfrak{t})+\sum_{\theta=1}^{\infty} \frac{(\Gamma(b))^{\theta} \mathfrak{t}^{(\theta-1)(a-1)}}{\Gamma(\theta b)} & \\ \times \sum_{\eta=0}^{\theta} C_{\eta}^{\theta} g_{2}^{\theta-\eta}(\mathfrak{t}) g_{3}^{\eta}(\mathfrak{t}) & a \in[1, \infty), b \in(0,1) . \\ \times \int_{0}^{\mathfrak{t}}(\mathfrak{t}-p)^{\theta b-1} p^{a-1} g_{1}(p) d p, & \end{cases}
$$

Proof. The proof of the inequality (1) would be followed by two cases. In the first case, we may assume $a, b \in(0,1)$ and in the second case, we may assume that $a \in$ $[1, \infty)$ and $b \in(0,1)$.

On letting

$$
\mathfrak{A} r(\mathfrak{t}):=g_{2}(\mathfrak{t}) \int_{0}^{\mathfrak{t}}(\mathfrak{t}-p)^{b-1} p^{a-1} r(p) d p+g_{3}(\mathfrak{t}) \int_{0}^{\mathfrak{t}} \mathfrak{t}^{b-1} p^{a-1} r(p) d p .
$$

In this case, (1) is reshaped as:

$$
r(\mathfrak{t}) \leq g_{1}(\mathfrak{t})+\mathfrak{A} r(\mathfrak{t}) .
$$


Iterating the inequality for some $\theta \in N$, one has

$$
r(\mathfrak{t}) \leq \sum_{\eta=0}^{\theta-1} \mathfrak{A}^{\eta} g_{1}(\mathfrak{t})+\mathfrak{A}^{\theta} r(\mathfrak{t}) .
$$

We claim that the following inequality does hold:

$$
\mathfrak{A}^{\theta} r(\mathfrak{t}) \leq \begin{cases}(\Gamma(b))^{\theta-1} \prod_{i=1}^{\theta-1} \frac{\Gamma(i c)}{\Gamma(i c+b)} \\ \\ \times \sum_{\eta=0}^{\theta} C_{\eta}^{\theta} g_{2}^{\theta-\eta}(\mathfrak{t}) g_{3}^{\eta}(\mathfrak{t}) \\ \times \int_{0}^{\mathfrak{t}}(\mathfrak{t}-p)^{\theta c-a} p^{a-1} r(p) d p, \quad a, b \in(0,1), \\ \frac{(\Gamma(b))^{\theta} \mathfrak{t}^{(\theta-1)(a-1)}}{\Gamma(\theta b)} \\ \times \sum_{\eta=0}^{\theta} C_{\eta}^{\theta} g_{2}^{\theta-\eta}(\mathfrak{t}) g_{3}^{\eta}(\mathfrak{t}) \\ \times \int_{0}^{\mathfrak{t}}(\mathfrak{t}-p)^{\theta b-1} p^{a-1} r(p) d p, \quad a \in[1, \infty), b \in(0,1),\end{cases}
$$

for some $\theta \in N$, where $\prod_{i=1}^{0} g(i)=1$.

Case-I: The proof follows the induction criteria on $\theta$. For $\theta=1$, consider

$$
\begin{aligned}
\mathfrak{A} r(\mathfrak{t}) & =g_{2}(\mathfrak{t}) \int_{0}^{\mathfrak{t}}(\mathfrak{t}-p)^{b-1} p^{a-1} r(p) d p+g_{3}(\mathfrak{t}) \int_{0}^{\mathfrak{t}} \mathfrak{t}^{b-1} p^{a-1} r(p) d p \\
& \leq\left(g_{2}(\mathfrak{t})+g_{3}(\mathfrak{t})\right) \int_{0}^{\mathfrak{t}}(\mathfrak{t}-p)^{b-1} p^{a-1} r(p) d p,
\end{aligned}
$$

which is true by virtue of $\prod_{i=1}^{0} g(i)=1$.

Suppose it holds for some $\theta=m$. Then, for $\theta=m+1$

$$
\begin{aligned}
\mathfrak{A}^{m+1} r(\mathfrak{t})= & \mathfrak{A}\left(\mathfrak{A}^{m} r(\mathfrak{t})\right) \\
= & g_{2}(\mathfrak{t}) \int_{0}^{\mathfrak{t}}(\mathfrak{t}-p)^{b-1} p^{a-1} \mathfrak{A}^{m} r(p) d p \\
& +g_{3}(\mathfrak{t}) \int_{0}^{\mathfrak{t}} \mathfrak{t}^{b-1} p^{a-1} \mathfrak{A}^{m} r(p) d p \\
\leq & g_{2}(\mathfrak{t}) \int_{0}^{\mathfrak{t}}(\mathfrak{t}-p)^{b-1} p^{a-1}(\Gamma(b))^{m-1} \prod_{i=1}^{m-1} \frac{\Gamma(i c)}{\Gamma(i c+b)} \\
& \times \sum_{\eta=0}^{m} C_{\eta}^{m} g_{2}^{m-\eta}(p) g_{3}^{\eta}(p) \int_{0}^{p}(p-\zeta)^{m c-a} \zeta^{a-1} r(\zeta) d \zeta d p \\
& +g_{3}(\mathfrak{t}) \int_{0}^{\mathfrak{t}} \mathfrak{t}^{b-1} p^{a-1}(\Gamma(b))^{m-1} \prod_{i=1}^{m-1} \frac{\Gamma(i c)}{\Gamma(i c+b)} \\
& \times \sum_{\eta=0}^{m} C_{\eta}^{m} g_{2}^{m-\eta}(p) g_{3}^{\eta}(p) \int_{0}^{p}(p-\zeta)^{m c-a} \zeta^{a-1} r(\zeta) d \zeta d p \\
\leq & (\Gamma(b))^{m-1} \prod_{i=1}^{m-1} \frac{\Gamma(i c)}{\Gamma(i c+b)} \sum_{\eta=0}^{m} C_{\eta}^{m} g_{2}^{m-\eta+1}(\mathfrak{t}) g_{3}^{\eta}(\mathfrak{t})
\end{aligned}
$$




$$
\begin{aligned}
& \times \int_{0}^{\mathfrak{t}}(\mathfrak{t}-p)^{b-1} p^{a-1} \int_{0}^{p}(p-\zeta)^{m c-a} \zeta^{a-1} r(\zeta) d \zeta d p \\
& +(\Gamma(b))^{m-1} \prod_{i=1}^{m-1} \frac{\Gamma(i c)}{\Gamma(i c+b)} \sum_{\eta=0}^{m} C_{\eta}^{m} g_{2}^{m-\eta}(\mathfrak{t}) g_{3}^{\eta+1}(\mathfrak{t}) \\
& \times \int_{0}^{\mathfrak{t}} \mathfrak{t}^{b-1} p^{a-1} \int_{0}^{p}(p-\zeta)^{m c-a} \zeta^{a-1} r(\zeta) d \zeta d p .
\end{aligned}
$$

Change of order of integration yields the following:

$$
\begin{aligned}
& \mathfrak{A}^{m+1} r(\mathfrak{t}) \leq(\Gamma(b))^{m-1} \prod_{i=1}^{m-1} \frac{\Gamma(i c)}{\Gamma(i c+b)} \sum_{\eta=0}^{m} C_{\eta}^{m} g_{2}^{m-\eta+1}(\mathfrak{t}) g_{3}^{\eta}(\mathfrak{t}) \\
& \times \int_{0}^{\mathfrak{t}} \zeta^{a-1} r(\zeta) \int_{\zeta}^{\mathfrak{t}}(\mathfrak{t}-p)^{b-1} p^{a-1}(p-\zeta)^{m c-a} d p d \zeta \\
& +(\Gamma(b))^{m-1} \prod_{i=1}^{m-1} \frac{\Gamma(i c)}{\Gamma(i c+b)} \sum_{\eta=0}^{m} C_{\eta}^{m} g_{2}^{m-\eta}(\mathfrak{t}) g_{3}^{\eta+1}(\mathfrak{t}) \\
& \times \int_{0}^{\mathfrak{t}} \zeta^{a-1} r(\zeta) \int_{\zeta}^{\mathfrak{t}}(\mathfrak{t}-p)^{b-1} p^{a-1}(p-\zeta)^{m c-a} d p d \zeta \\
& \leq(\Gamma(b))^{m-1} \prod_{i=1}^{m-1} \frac{\Gamma(i c)}{\Gamma(i c+b)} \sum_{\eta=0}^{m} C_{\eta}^{m} g_{2}^{m-\eta+1}(\mathfrak{t}) g_{3}^{\eta}(\mathfrak{t}) \\
& \times \int_{0}^{\mathfrak{t}} \zeta^{a-1} r(\zeta) \int_{\zeta}^{\mathfrak{t}}(\mathfrak{t}-p)^{b-1}(p-\zeta)^{m c-1} d p d \zeta \\
& +(\Gamma(b))^{m-1} \prod_{i=1}^{m-1} \frac{\Gamma(i c)}{\Gamma(i c+b)} \sum_{\eta=0}^{m} C_{\eta}^{m} g_{2}^{m-\eta}(\mathfrak{t}) g_{3}^{\eta+1}(\mathfrak{t}) \\
& \times \int_{0}^{\mathfrak{t}} \zeta^{a-1} r(\zeta) \int_{\zeta}^{\mathfrak{t}}(\mathfrak{t}-p)^{b-1}(p-\zeta)^{m c-1} d p d \zeta \\
& =(\Gamma(b))^{m-1} \prod_{i=1}^{m-1} \frac{\Gamma(i c)}{\Gamma(i c+b)} \sum_{\eta=0}^{m} C_{\eta}^{m} g_{2}^{m-\eta+1}(\mathfrak{t}) g_{3}^{\eta}(\mathfrak{t}) \\
& \times \int_{0}^{\mathfrak{t}} \zeta^{a-1} r(\zeta) \frac{\Gamma(b) \Gamma(m c)}{\Gamma(b+m c)}(\mathfrak{t}-\zeta)^{b+m c-1} d \zeta \\
& +(\Gamma(b))^{m-1} \prod_{i=1}^{m-1} \frac{\Gamma(i c)}{\Gamma(i c+b)} \sum_{\eta=0}^{m} C_{\eta}^{m} g_{2}^{m-\eta}(\mathfrak{t}) g_{3}^{\eta+1}(\mathfrak{t}) \\
& \times \int_{0}^{\mathfrak{t}} \zeta^{a-1} r(\zeta) \frac{\Gamma(b) \Gamma(m c)}{\Gamma(b+m c)}(\mathfrak{t}-\zeta)^{b+m c-1} d \zeta \\
& =(\Gamma(b))^{m} \prod_{i=1}^{m} \frac{\Gamma(i c)}{\Gamma(i c+b)} \sum_{\eta=0}^{m} C_{\eta}^{m} g_{2}^{m-\eta+1}(\mathfrak{t}) g_{3}^{\eta}(\mathfrak{t}) \\
& \times \int_{0}^{\mathfrak{t}}(\mathfrak{t}-\zeta)^{(m+1) c-a} \zeta^{a-1} r(\zeta) d \zeta
\end{aligned}
$$




$$
\begin{aligned}
& +(\Gamma(b))^{m} \prod_{i=1}^{m} \frac{\Gamma(i c)}{\Gamma(i c+b)} \sum_{\eta=0}^{m} C_{\eta}^{m} g_{2}^{m-\eta}(\mathfrak{t}) g_{3}^{\eta+1}(\mathfrak{t}) \\
& \times \int_{0}^{\mathfrak{t}}(\mathfrak{t}-\zeta)^{(m+1) c-a} \zeta^{a-1} r(\zeta) d \zeta \\
& =(\Gamma(b))^{m} \prod_{i=1}^{m} \frac{\Gamma(i c)}{\Gamma(i c+b)} C_{0}^{m} g_{2}^{m+1}(\mathfrak{t}) \int_{0}^{\mathfrak{t}}(\mathfrak{t}-\zeta)^{(m+1) c-a} \zeta^{a-1} r(\zeta) d \zeta \\
& +(\Gamma(b))^{m} \prod_{i=1}^{m} \frac{\Gamma(i c)}{\Gamma(i c+b)} \sum_{\eta=1}^{m} C_{\eta}^{m} g_{2}^{m-\eta+1}(\mathfrak{t}) g_{3}^{\eta}(\mathfrak{t}) \\
& \times \int_{0}^{\mathfrak{t}}(\mathfrak{t}-\zeta)^{(m+1) c-a} \zeta^{a-1} r(\zeta) d \zeta \\
& +(\Gamma(b))^{m} \prod_{i=1}^{m} \frac{\Gamma(i c)}{\Gamma(i c+b)} \sum_{\eta=1}^{m} C_{\eta-1}^{m} g_{2}^{m-\eta+1}(\mathfrak{t}) g_{3}^{\eta}(\mathfrak{t}) \\
& \times \int_{0}^{\mathfrak{t}}(\mathfrak{t}-\zeta)^{(m+1) c-a} \zeta^{a-1} r(\zeta) d \zeta \\
& +(\Gamma(b))^{m} \prod_{i=1}^{m} \frac{\Gamma(i c)}{\Gamma(i c+b)} C_{m}^{m} g_{3}^{m+1}(\mathfrak{t}) \int_{0}^{\mathfrak{t}}(\mathfrak{t}-\zeta)^{(m+1) c-a} \zeta^{a-1} r(\zeta) d \zeta \\
& =(\Gamma(b))^{m} \prod_{i=1}^{m} \frac{\Gamma(i c)}{\Gamma(i c+b)} C_{0}^{m+1} g_{2}^{m+1}(\mathfrak{t}) \int_{0}^{\mathfrak{t}}(\mathfrak{t}-\zeta)^{(m+1) c-a} \zeta^{a-1} r(\zeta) d \zeta \\
& +(\Gamma(b))^{m} \prod_{i=1}^{m} \frac{\Gamma(i c)}{\Gamma(i c+b)} \sum_{\eta=1}^{m}\left(C_{\eta}^{m}+C_{\eta-1}^{m}\right) g_{2}^{m-\eta+1}(\mathfrak{t}) g_{3}^{\eta}(\mathfrak{t}) \\
& \times \int_{0}^{\mathfrak{t}}(\mathfrak{t}-\zeta)^{(m+1) c-a} \zeta^{a-1} r(\zeta) d \zeta \\
& +(\Gamma(b))^{m} \prod_{i=1}^{m} \frac{\Gamma(i c)}{\Gamma(i c+b)} C_{m+1}^{m+1} g_{3}^{m+1}(\mathfrak{t}) \int_{0}^{\mathfrak{t}}(\mathfrak{t}-\zeta)^{(m+1) c-a} \zeta^{a-1} r(\zeta) d \zeta \\
& =(\Gamma(b))^{m} \prod_{i=1}^{m} \frac{\Gamma(i c)}{\Gamma(i c+b)} \sum_{\eta=0}^{m+1} C_{\eta}^{m+1} g_{2}^{m-\eta+1}(\mathfrak{t}) g_{3}^{\eta}(\mathfrak{t}) \\
& \times \int_{0}^{\mathfrak{t}}(\mathfrak{t}-\zeta)^{(m+1) c-a} \zeta^{a-1} r(\zeta) d \zeta,
\end{aligned}
$$

which is no more than inequality (4) for $\theta=m+1$.

Case-II: For $\theta=1$, the steps are same as $a, b \in(0,1)$.

Suppose (4) holds for some $\theta=m$. Then, for $\theta=m+1$, consider

$$
\begin{aligned}
\mathfrak{A}^{m+1} r(\mathfrak{t}) & =\mathfrak{A}\left(\mathfrak{A}^{m} r(\mathfrak{t})\right) \\
& =g_{2}(\mathfrak{t}) \int_{0}^{\mathfrak{t}}(\mathfrak{t}-p)^{b-1} p^{a-1} \mathfrak{A}^{m} r(p) d p+g_{3}(\mathfrak{t}) \int_{0}^{\mathfrak{t}} \mathfrak{t}^{b-1} p^{a-1} \mathfrak{A}^{m} r(p) d p \\
& \leq g_{2}(\mathfrak{t}) \int_{0}^{\mathfrak{t}}(\mathfrak{t}-p)^{b-1} p^{a-1} \frac{(\Gamma(b))^{m} p^{(m-1)(a-1)}}{\Gamma(m b)} \sum_{\eta=0}^{m} C_{\eta}^{m} g_{2}^{m-\eta}(p) g_{3}^{\eta}(p)
\end{aligned}
$$




$$
\begin{aligned}
& \times \int_{0}^{p}(p-\zeta)^{m b-1} \zeta^{a-1} r(\zeta) d \zeta d p+g_{3}(\mathfrak{t}) \int_{0}^{\mathfrak{t}} \mathfrak{t}^{b-1} p^{a-1} \\
& \times \frac{(\Gamma(b))^{m} p^{(m-1)(a-1)}}{\Gamma(m b)} \\
& \times \sum_{\eta=0}^{m} C_{\eta}^{m} g_{2}^{m-\eta}(p) g_{3}^{\eta}(p) \int_{0}^{p}(p-\zeta)^{m b-1} \zeta^{a-1} r(\zeta) d \zeta d p \\
& \leq \frac{(\Gamma(b))^{m}}{\Gamma(m b)} \sum_{\eta=0}^{m} C_{\eta}^{m} g_{2}^{m-\eta+1}(\mathfrak{t}) g_{3}^{\eta}(\mathfrak{t}) \int_{0}^{\mathfrak{t}}(\mathfrak{t}-p)^{b-1} p^{a-1} p^{(m-1)(a-1)} \\
& \times \int_{0}^{p}(p-\zeta)^{m b-1} \zeta^{a-1} r(\zeta) d \zeta d p \\
& +\frac{(\Gamma(b))^{m}}{\Gamma(m b)} \sum_{\eta=0}^{m} C_{\eta}^{m} g_{2}^{m-\eta}(\mathfrak{t}) g_{3}^{\eta+1}(\mathfrak{t}) \\
& \times \int_{0}^{\mathfrak{t}} \mathfrak{t}^{b-1} p^{a-1} p^{(m-1)(a-1)} \int_{0}^{p}(p-\zeta)^{m b-1} \zeta^{a-1} r(\zeta) d \zeta d p \\
& \leq \frac{(\Gamma(b))^{m} \mathfrak{t}^{m(a-1)}}{\Gamma(m b)} \sum_{\eta=0}^{m} C_{\eta}^{m} g_{2}^{m-\eta+1}(\mathfrak{t}) g_{3}^{\eta}(\mathfrak{t}) \int_{0}^{\mathfrak{t}}(\mathfrak{t}-p)^{b-1} \\
& \times \int_{0}^{p}(p-\zeta)^{m b-1} \zeta^{a-1} r(\zeta) d \zeta d p+\frac{(\Gamma(b))^{m} \mathfrak{t}^{m(a-1)}}{\Gamma(m b)} \\
& \times \sum_{\eta=0}^{m} C_{\eta}^{m} g_{2}^{m-\eta}(\mathfrak{t}) g_{3}^{\eta+1}(\mathfrak{t}) \int_{0}^{\mathfrak{t}} \mathfrak{t}^{b-1} \int_{0}^{p}(p-\zeta)^{m b-1} \zeta^{a-1} r(\zeta) d \zeta d p .
\end{aligned}
$$

Interchanging the order of integration yields

$$
\begin{aligned}
\mathfrak{A}^{m+1} r(\mathfrak{t}) \leq & \frac{(\Gamma(b))^{m} \mathfrak{t}^{m(a-1)}}{\Gamma(m b)} \sum_{\eta=0}^{m} C_{\eta}^{m} g_{2}^{m-\eta+1}(\mathfrak{t}) g_{3}^{\eta}(\mathfrak{t}) \\
& \times \int_{0}^{\mathfrak{t}} \zeta^{a-1} r(\zeta) \int_{\zeta}^{\mathfrak{t}}(\mathfrak{t}-p)^{b-1}(p-\zeta)^{m b-1} d p d \zeta \\
& +\frac{(\Gamma(b))^{m} \mathfrak{t}^{m(a-1)}}{\Gamma(m b)} \sum_{\eta=0}^{m} C_{\eta}^{m} g_{2}^{m-\eta}(\mathfrak{t}) g_{3}^{\eta+1}(\mathfrak{t}) \\
& \times \int_{0}^{\mathfrak{t}} \zeta^{a-1} r(\zeta) \int_{\zeta}^{\mathfrak{t}}(\mathfrak{t}-p)^{b-1}(p-\zeta)^{m b-1} d p d \zeta \\
= & \frac{(\Gamma(b))^{m} \mathfrak{t}^{m(a-1)} \sum_{\eta=0}^{m} C_{\eta}^{m} g_{2}^{m-\eta+1}(\mathfrak{t}) g_{3}^{\eta}(\mathfrak{t}) \int_{0}^{\mathfrak{t}} \zeta^{a-1} r(\zeta)}{\Gamma(\zeta)} \\
& \times \frac{\Gamma(b) \Gamma(m b)}{\Gamma(b+m b)}(\mathfrak{t}-\zeta)^{b+m b-1} d \zeta+\frac{(\Gamma(b))^{m} \mathfrak{t}^{m(a-1)}}{\Gamma(m b)} \\
& \times \sum_{\eta=0}^{m} C_{\eta}^{m} g_{2}^{m-\eta}(\mathfrak{t}) g_{3}^{\eta+1}(\mathfrak{t}) \int_{0}^{\mathfrak{t}} \zeta^{a-1} r(\zeta)
\end{aligned}
$$




$$
\begin{aligned}
& \times \frac{\Gamma(b) \Gamma(m b)}{\Gamma(b+m b)}(\mathfrak{t}-\zeta)^{b+m b-1} d \zeta \\
= & \frac{(\Gamma(b))^{m+1} \mathfrak{t}^{m(a-1)}}{\Gamma((m+1) b)} \sum_{\eta=0}^{m} C_{\eta}^{m} g_{2}^{m-\eta+1}(\mathfrak{t}) g_{3}^{\eta}(\mathfrak{t}) \\
& \times \int_{0}^{\mathfrak{t}}(\mathfrak{t}-\zeta)^{(m+1) b-1} \zeta^{a-1} r(\zeta) d \zeta \\
& +\frac{(\Gamma(b))^{m+1} \mathfrak{t}^{m(a-1)}}{\Gamma((m+1) b)} \sum_{\eta=0}^{m} C_{\eta}^{m} g_{2}^{m-\eta}(\mathfrak{t}) g_{3}^{\eta+1}(\mathfrak{t}) \\
& \times \int_{0}^{\mathfrak{t}}(\mathfrak{t}-\zeta)^{(m+1) b-1} \zeta^{a-1} r(\zeta) d \zeta \\
= & \frac{(\Gamma(b))^{m+1} \mathfrak{t}^{m(a-1)}}{\Gamma((m+1) b)} \sum_{\eta=0}^{m+1} C_{\eta}^{m+1} g_{2}^{m-\eta+1}(\mathfrak{t}) g_{3}^{\eta}(\mathfrak{t}) \\
& \times \int_{0}^{\mathfrak{t}}(\mathfrak{t}-\zeta)^{(m+1) b-1} \zeta^{a-1} r(\zeta) d \zeta
\end{aligned}
$$

which is no more than inequality (4) for $\theta=m+1$. We further, claim that $\mathfrak{A}^{\theta} r(\mathfrak{t}) \rightarrow 0$ as $\theta \rightarrow \infty$. Now, we go back to inequality (4).

For the case $a, b \in(0,1)$, there exists $N_{1}>0$ such that for $\theta>N_{1}$, we have

$$
\theta c-a>0
$$

and hence for an arbitrary $\omega>0$

$$
(\mathfrak{t}-p)^{\theta c-a} \leq \omega^{\theta c-a}, \quad \mathfrak{t} \in[0, \omega], p \in[0, \mathfrak{t}] .
$$

Therefore, for $\theta>N_{1}$ and $\mathfrak{t} \in[0, \omega]$, we have

$$
\begin{aligned}
\mathfrak{A}^{\theta} r(\mathfrak{t}) & \leq(\Gamma(b))^{\theta-1} \prod_{i=1}^{\theta-1} \frac{\Gamma(i c)}{\Gamma(i c+b)} \sum_{\eta=0}^{\theta} C_{\eta}^{\theta} g_{2}^{\theta-\eta}(\mathfrak{t}) g_{3}^{\eta}(\mathfrak{t}) \int_{0}^{\mathfrak{t}}(\mathfrak{t}-p)^{\theta c-a} p^{a-1} r(p) d p \\
& \leq(\Gamma(b))^{\theta-1} \prod_{i=1}^{\theta-1} \frac{\Gamma(i c)}{\Gamma(i c+b)}\left(g_{2}(\mathfrak{t})+g_{3}(\mathfrak{t})\right)^{\theta} \int_{0}^{\mathfrak{t}} \omega^{\theta c-a} p^{a-1} r(p) d p \\
& \leq(\Gamma(b))^{\theta-1} \prod_{i=1}^{\theta-1} \frac{\Gamma(i c)}{\Gamma(i c+b)}\left(g_{2}(\mathfrak{t})+g_{3}(\mathfrak{t})\right)^{\theta} \omega^{\theta c-a} \int_{0}^{\omega} p^{a-1} r(p) d p
\end{aligned}
$$

For

$$
\mathfrak{B}_{\theta}:=(\Gamma(b))^{\theta-1} \prod_{i=1}^{\theta-1} \frac{\Gamma(i c)}{\Gamma(i c+b)}\left(g_{2}(\mathfrak{t})+g_{3}(\mathfrak{t})\right)^{\theta} \omega^{\theta c-a} .
$$

Since $g_{2}(\mathfrak{t})$ and $g_{3}(\mathfrak{t})$ are bounded, so by Lemma 1

$$
\frac{\mathfrak{B}_{\theta+1}}{\mathfrak{B}_{\theta}}=\frac{\Gamma(b) \Gamma(\theta c)}{\Gamma(\theta c+b)}\left(g_{2}(\mathfrak{t})+g_{3}(\mathfrak{t})\right) \omega^{c} \rightarrow 0 \quad \text { as } \theta \rightarrow \infty
$$


$p^{a-1} r(p)$ is locally integrable over $R^{+}$, so

$$
\mathfrak{A}^{\theta} r(\mathfrak{t}) \rightarrow 0 \text { as } \theta \rightarrow \infty
$$

Similarly, we can prove that for $\theta>N_{2}$ and $\mathfrak{t} \in[0, \omega]$,

$$
\begin{aligned}
\sum_{\theta=1}^{\infty} \mathfrak{A}^{\theta} g_{1}(\mathfrak{t}) & =\sum_{\theta=1}^{N_{2}} \mathfrak{A}^{\theta} g_{1}(\mathfrak{t})+\sum_{\theta=N_{2}+1}^{\infty} \mathfrak{A}^{\theta} g_{1}(\mathfrak{t}) \\
& \leq \sum_{\theta=1}^{N_{2}} \mathfrak{A}^{\theta} g_{1}(\mathfrak{t})+\sum_{\theta=N_{2}+1}^{\infty} \mathfrak{B}_{\theta} \int_{0}^{\omega} p^{a-1} r(p) d p \\
& <\infty .
\end{aligned}
$$

In a similar fashion, in Case-II, some one can prove $\mathfrak{A}^{\theta} r(\mathfrak{t}) \stackrel{\theta \rightarrow \infty}{\longrightarrow} 0$ and convergence of $\sum_{\theta=1}^{\infty} \mathfrak{A}^{\theta} g_{1}(\mathfrak{t})$ for $\mathfrak{t} \in[0, \omega]$.

For $g_{1}(\mathfrak{t})=g \mathfrak{t}^{d-1}$ in theorem 3 , the following holds.

Corollary 4. Let $a, d>0 ; 0<b<1 ; c=a+b-1>0 ; e=a+d-1>$ $0 ; g>0 ; g_{2}(\mathfrak{t})$ and $g_{3}(\mathfrak{t})$ are nonnegative, nondecreasing, bounded and continuous functions defined on $\mathbf{R}^{+}$. Further, suppose that $r(\mathfrak{t})$ is a nonnegative and $\mathfrak{t}^{a-1} r(\mathfrak{t})$ is locally integrable on $\mathbf{R}^{+}$such that:

$$
r(\mathfrak{t}) \leq g \mathfrak{t}^{d-1}+g_{2}(\mathfrak{t}) \int_{0}^{\mathfrak{t}}(\mathfrak{t}-p)^{b-1} p^{a-1} r(p) d p+g_{3}(\mathfrak{t}) \int_{0}^{\mathfrak{t}} \mathfrak{t}^{b-1} p^{a-1} r(p) d p .
$$

Then

$$
r(\mathfrak{t}) \leq g \mathfrak{t}^{d-1} F_{c, e, b+e}\left(\Gamma(b)\left(g_{2}(\mathfrak{t})+g_{3}(\mathfrak{t})\right) \mathfrak{t}^{c}\right), \quad \mathfrak{t} \in \mathbf{R}^{+} .
$$

Proof. From the proof of theorem 3, we have $\mathfrak{A}^{\theta} r(\mathfrak{t}) \rightarrow 0$ as $\theta \rightarrow \infty$ for the cases $a, b \in(0,1)$ and $a \in[1, \infty), b \in(0,1)$. This, together with (3), leads to

$$
r(\mathfrak{t}) \leq \sum_{\eta=0}^{\infty}\left(\mathfrak{A}^{\eta} g \mathfrak{t}^{d-1}\right)(\mathfrak{t})
$$

Now, we show that

$$
\left(\mathfrak{A}^{\eta} g \mathfrak{t}^{d-1}\right)(\mathfrak{t}) \leq g \mathfrak{t}^{d-1}\left(\mathfrak{t}^{c} \Gamma(b)\right)^{\eta} \prod_{i=0}^{\eta-1} \frac{\Gamma(i c+e)}{\Gamma(b+i c+e)} \sum_{i=0}^{\eta} C_{i}^{\eta} g_{2}^{\eta-i}(\mathfrak{t}) g_{3}^{i}(\mathfrak{t}),
$$

where $\eta \in N$.

For $\theta=0$, the result holds by virtue of $\prod_{i=0}^{\eta-1} g(i)=1$. Suppose it holds for some $\theta=\eta$. For $\theta=\eta+1$, one has

$$
\left(\mathfrak{A}^{\eta} g \mathfrak{t}^{d-1}\right)(\mathfrak{t})=g_{2}(\mathfrak{t}) \int_{0}^{\mathfrak{t}}(\mathfrak{t}-p)^{b-1} p^{a-1}\left(\mathfrak{A}^{\eta} g p^{d-1}\right)(p) d p
$$




$$
\begin{aligned}
& +g_{3}(\mathfrak{t}) \int_{0}^{\mathfrak{t}} \mathfrak{t}^{b-1} p^{a-1}\left(\mathfrak{A}^{\eta} g p^{d-1}\right)(p) d p \\
& \leq g_{2}(\mathfrak{t}) \int_{0}^{\mathfrak{t}}(\mathfrak{t}-p)^{b-1} p^{a-1} g p^{d-1}\left(p^{c} \Gamma(b)\right)^{\eta} \prod_{i=0}^{\eta-1} \frac{\Gamma(i c+e)}{\Gamma(b+i c+e)} \\
& \times \sum_{i=0}^{\eta} C_{i}^{\eta} g_{2}^{\eta-i}(p) g_{3}^{i}(p) d p+g_{3}(\mathfrak{t}) \int_{0}^{\mathfrak{t}} \mathfrak{t}^{b-1} p^{a-1} g p^{d-1}\left(p^{c} \Gamma(b)\right)^{\eta} \\
& \times \prod_{i=0}^{\eta-1} \frac{\Gamma(i c+e)}{\Gamma(b+i c+e)} \sum_{i=0}^{\eta} C_{i}^{\eta} g_{2}^{\eta-i}(p) g_{3}^{i}(p) d p \\
& \leq g(\Gamma(b))^{\eta} \prod_{i=0}^{\eta-1} \frac{\Gamma(i c+e)}{\Gamma(b+i c+e)} \sum_{i=0}^{\eta} C_{i}^{\eta} g_{2}^{\eta-i+1}(\mathfrak{t}) g_{3}^{i}(\mathfrak{t}) \\
& \times \int_{0}^{\mathfrak{t}}(\mathfrak{t}-p)^{b-1} p^{a-1} p^{d-1} p^{\eta c} d p+g(\Gamma(b))^{\eta} \prod_{i=0}^{\eta-1} \frac{\Gamma(i c+e)}{\Gamma(b+i c+e)} \\
& \times \sum_{i=0}^{\eta} C_{i}^{\eta} g_{2}^{\eta-i}(\mathfrak{t}) g_{3}^{i+1}(\mathfrak{t}) \int_{0}^{\mathfrak{t}} \mathfrak{t}^{b-1} p^{a-1} p^{d-1} p^{\eta c} d p \\
& \leq g(\Gamma(b))^{\eta} \prod_{i=0}^{\eta-1} \frac{\Gamma(i c+e)}{\Gamma(b+i c+e)} \sum_{i=0}^{\eta+1} C_{i}^{\eta+1} g_{2}^{\eta-i+1}(\mathfrak{t}) g_{3}^{i}(\mathfrak{t}) \\
& \times \int_{0}^{\mathfrak{t}}(\mathfrak{t}-p)^{b-1} p^{a+d+\eta c-2} d p \\
& =g(\Gamma(b))^{\eta} \prod_{i=0}^{\eta-1} \frac{\Gamma(i c+e)}{\Gamma(b+i c+e)} \sum_{i=0}^{\eta+1} C_{i}^{\eta+1} g_{2}^{\eta-i+1}(\mathfrak{t}) g_{3}^{i}(\mathfrak{t}) \\
& \times \frac{\Gamma(b) \Gamma(a+d+\eta c-1)}{\Gamma(a+b+d+\eta c-1)} \mathfrak{t}^{a+b+d+\eta c-2} \\
& =g \mathfrak{t}^{d-1}\left(\mathfrak{t}^{c} \Gamma(b)\right)^{\eta+1} \prod_{i=0}^{\eta} \frac{\Gamma(i c+e)}{\Gamma(b+i c+e)} \sum_{i=0}^{\eta+1} C_{i}^{\eta+1} g_{2}^{\eta-i+1}(\mathfrak{t}) g_{3}^{i}(\mathfrak{t}) .
\end{aligned}
$$

Hence, inequality (8) is satisfied for any $\eta \in N$. In other words, we have proved that

$$
r(\mathfrak{t}) \leq \sum_{\eta=0}^{\infty} g \mathfrak{t}^{d-1}\left(\mathfrak{t}^{c} \Gamma(b)\right)^{\eta} \prod_{i=0}^{\eta-1} \frac{\Gamma(i c+e)}{\Gamma(b+i c+e)} \sum_{i=0}^{\eta} C_{i}^{\eta} g_{2}^{\eta-i}(\mathfrak{t}) g_{3}^{i}(\mathfrak{t}) .
$$

By definition 2

$$
r(\mathfrak{t}) \leq g \mathfrak{t}^{d-1} F_{c, e, b+e}\left(\Gamma(b)\left(g_{2}(\mathfrak{t})+g_{3}(\mathfrak{t})\right) \mathfrak{t}^{c}\right)
$$

Remark 5. For $g_{3}(\mathfrak{t}) \equiv 0, \mathfrak{t}>0$, Corollary 4 reduces to [4, Theorem 2.7] for $b \in(0,1)$. 


\section{APPLICATION}

Consider the following stochastic differential equation:

$$
d(x(\mathfrak{t}))=b(\mathfrak{t}, x(\mathfrak{t})) d \mathfrak{t}+\sigma_{1}(\mathfrak{t}, x(\mathfrak{t})) d \mathfrak{t}^{a}+\sigma_{2}(\mathfrak{t}, x(\mathfrak{t})) d B_{\mathfrak{t}}
$$

where $0<a<1$ and $B_{\mathfrak{t}}$ is the standard Brownian motion.

Theorem 6. Let $\omega>0 ; a \in(0,1) ;(\Omega, F, P)$ be a complete probability space with an $m$-dimensional Brownian motion $B(\mathfrak{t})$ defined on space $\mathbb{R}^{n}$; let $w_{0}$ be a random variable such that $E\left|w_{0}\right|^{2}<\infty$; let $b(.,),. \sigma_{1}(.,):.[0, \omega] \times \mathbb{R}^{n} \rightarrow \mathbb{R}^{n}$ and $\sigma_{2}(.,):.[0, \omega] \times \mathbb{R}^{n} \rightarrow \mathbb{R}^{n \times m}$ be measurable functions such that $\mathfrak{t}^{1-a} b(.,),. \mathfrak{t}^{1-a} \sigma_{1}(.,$.$) ,$ $\mathfrak{t}^{1-a} \sigma_{2}(.,$.$) are also measurable such that the linear Growth and Lipschitz conditions,$

$$
\begin{gathered}
|b(\mathfrak{t}, x)|^{2}+\left|\sigma_{1}(\mathfrak{t}, x)\right|^{2}+\left|\sigma_{2}(\mathfrak{t}, x)\right|^{2} \leq K^{2}\left(1+|x|^{2}\right) \\
|b(\mathfrak{t}, x)-b(\mathfrak{t}, y)|+\left|\sigma_{1}(\mathfrak{t}, x)-\sigma_{1}(\mathfrak{t}, y)\right|+\left|\sigma_{2}(\mathfrak{t}, x)-\sigma_{2}(\mathfrak{t}, y)\right| \leq L|x-y|
\end{gathered}
$$

are satisfied, for some constants $K, L>0$. Then the fractional stochastic differential equation (9) has a $t$-continuous solution with a filtration $F_{t}^{w_{0}}$ such that

$$
E\left[\int_{0}^{\omega}|x(\mathfrak{t})|^{2} d \mathfrak{t}\right]<\infty .
$$

Proof. The integral form of the stochastic differential equation (9) is

$$
\begin{aligned}
x(\mathfrak{t})= & w_{0}+\int_{0}^{\mathfrak{t}} b(p, x(p)) d p+a \int_{0}^{\mathfrak{t}}(\mathfrak{t}-p)^{a-1} \sigma_{1}(p, x(p)) d p \\
& +\int_{0}^{\mathfrak{t}} \sigma_{2}(p, x(p)) d B_{p} .
\end{aligned}
$$

By the method of Picard-Lindelöf iteration, define logarithmically $x^{(0)}(\mathfrak{t})=x_{0}$, for some $\eta \in N$, as follows:

$$
\begin{aligned}
x^{(\eta+1)}(\mathfrak{t})= & w_{0}+\int_{0}^{\mathfrak{t}} b\left(p, x^{(\eta)}(p)\right) d p+a \int_{0}^{\mathfrak{t}}(\mathfrak{t}-p)^{a-1} \sigma_{1}\left(p, x^{(\eta)}(p)\right) d p \\
& +\int_{0}^{\mathfrak{t}} \sigma_{2}\left(p, x^{(\eta)}(p)\right) d B_{p} .
\end{aligned}
$$

Using the inequality $|x+y+z|^{2} \leq 3|x|^{2}+3|y|^{2}+3|z|^{2}$, we have

$$
\begin{aligned}
& E\left|x^{(\eta+1)}(\mathfrak{t})-x^{(\eta)}(\mathfrak{t})\right|^{2} \\
\leq & 3 E\left|\int_{0}^{\mathfrak{t}}\left\{b\left(p, x^{(\eta)}(p)\right)-b\left(p, x^{(\eta-1)}(p)\right)\right\} d p\right|^{2}
\end{aligned}
$$




$$
\begin{aligned}
& +3 E\left|a \int_{0}^{\mathfrak{t}}(\mathfrak{t}-p)^{a-1}\left\{\sigma_{1}\left(p, x^{(\eta)}(p)\right)-\sigma_{1}\left(p, x^{(\eta-1)}(p)\right)\right\} d p\right|^{2} \\
& +3 E\left|\int_{0}^{\mathfrak{t}}\left\{\sigma_{2}\left(p, x^{(\eta)}(p)\right)-\sigma_{2}\left(p, x^{(\eta-1)}(p)\right)\right\} d B_{p}\right|^{2} .
\end{aligned}
$$

Cauchy Schwartz inequality on the first two integral and Itô's Isometry on the third integral yields:

$$
\begin{aligned}
& E\left|x^{(\eta+1)}(\mathfrak{t})-x^{(\eta)}(\mathfrak{t})\right|^{2} \\
\leq & 3 \omega E \int_{0}^{\mathfrak{t}}\left[b\left(p, x^{(\eta)}(p)\right)-b\left(p, x^{(\eta-1)}(p)\right)\right]^{2} d p \\
& +3 a \mathfrak{t}^{a} E \int_{0}^{\mathfrak{t}}(\mathfrak{t}-p)^{a-1}\left[\sigma_{1}\left(p, x^{(\eta)}(p)\right)-\sigma_{1}\left(p, x^{(\eta-1)}(p)\right)\right]^{2} d p \\
+ & 3 E \int_{0}^{\mathfrak{t}}\left[\sigma_{2}\left(p, x^{(\eta)}(p)\right)-\sigma_{2}\left(p, x^{(\eta-1)}(p)\right)\right]^{2} d p .
\end{aligned}
$$

Application of the Lipschitz codition (11) yields:

$$
\begin{aligned}
E \mid x^{(\eta+1)}(\mathfrak{t})- & \left.x^{(\eta)}(\mathfrak{t})\right|^{2} \leq 3 L^{2}(1+\omega) \int_{0}^{\mathfrak{t}} E\left|x^{(\eta)}(p)-x^{(\eta-1)}(p)\right|^{2} d p \\
& +3 L^{2}(1+\omega) \int_{0}^{\mathfrak{t}}(\mathfrak{t}-p)^{a-1} E\left|x^{(\eta)}(p)-x^{(\eta-1)}(p)\right|^{2} d p \\
\Rightarrow & \mathfrak{t}^{1-a} E\left|x^{(\eta+1)}(\mathfrak{t})-x^{(\eta)}(\mathfrak{t})\right|^{2} \leq 3 L^{2}(1+\omega) \omega^{1-a} \times\left[\omega^{1-a}\right. \\
& \times \int_{0}^{\mathfrak{t}} \mathfrak{t}^{a-1} p^{a-1}\left\{p^{1-a} E\left|x^{(\eta)}(p)-x^{(\eta-1)}(p)\right|^{2}\right\} d p+ \\
& \left.\quad \int_{0}^{\mathfrak{t}}(\mathfrak{t}-p)^{a-1} p^{a-1}\left\{p^{1-a} E\left|x^{(\eta)}(p)-x^{(\eta-1)}(p)\right|^{2}\right\} d p\right] .
\end{aligned}
$$

For locally integrable function $\Psi(\mathfrak{t})$ define an operator $\mathfrak{C}$ as follows:

$$
\begin{aligned}
\mathfrak{C} \Psi(\mathfrak{t}):= & 3 L^{2}(1+\omega) \omega^{1-a}\left[\omega^{1-a} \int_{0}^{\mathfrak{t}} \mathfrak{t}^{a-1} p^{a-1} \Psi(p) d p\right. \\
& \left.+\int_{0}^{t}(\mathfrak{t}-p)^{a-1} p^{a-1} \Psi(p) d p\right] .
\end{aligned}
$$

From (16) and (17), repeating iteration yields:

$$
\begin{aligned}
& \mathfrak{t}^{1-a} E\left|x^{(\eta+1)}(\mathfrak{t})-x^{(\eta)}(\mathfrak{t})\right|^{2} \leq \mathfrak{C}\left(\mathfrak{t}^{1-a} E\left|x^{(\eta)}(\mathfrak{t})-x^{(\eta-1)}(\mathfrak{t})\right|^{2}\right) \\
& \leq \ldots \leq \mathfrak{C}^{\eta-1}\left(\mathfrak{t}^{1-a} E\left|x^{(2)}(\mathfrak{t})-x^{(1)}(\mathfrak{t})\right|^{2}\right) \leq \mathfrak{C}^{\eta}\left(\mathfrak{t}^{1-a} E\left|x^{(1)}(\mathfrak{t})-x^{(0)}(\mathfrak{t})\right|^{2}\right)
\end{aligned}
$$


As, $E\left|x^{(1)}(\mathfrak{t})-x^{(0)}(\mathfrak{t})\right|^{2}$ is locally integrable therefore application of (4), (5) and (18) yield:

$$
\begin{aligned}
\mathfrak{t}^{1-a} E\left|x^{(\eta+1)}(\mathfrak{t})-x^{(\eta)}(\mathfrak{t})\right|^{2} \leq & \mathfrak{C}^{\eta}\left(\mathfrak{t}^{1-a} E\left|x^{(1)}(\mathfrak{t})-x^{(0)}(\mathfrak{t})\right|^{2}\right) \\
\leq & (\Gamma(a))^{\eta-1} \prod_{i=1}^{\eta-1} \frac{\Gamma(i(2 a-1))}{\Gamma(i(2 a-1)+a)} \omega^{-a} \\
& \times\left[3 L^{2}(1+\omega) \omega^{a}\left(1+\omega^{1-a}\right)\right]^{\eta} \\
& \times \int_{0}^{\mathfrak{t}} E\left|x^{(1)}(p)-x^{(0)}(p)\right|^{2} d p .
\end{aligned}
$$

Again, from (13) applications of the inequality $|x+y+z|^{2} \leq 3|x|^{2}+3|y|^{2}+3|z|^{2}$, Cauchy Schwartz inequality, Itô's Isometry, linear growth condition yields

$$
E\left|x^{(1)}(\mathfrak{t})-x^{(0)}(\mathfrak{t})\right|^{2} \leq 3 K^{2}\left(1+E\left|w_{0}\right|^{2}\right)(1+\omega)\left(\mathfrak{t}+\mathfrak{t}^{a}\right) .
$$

A Combination of (19) and (20) produces

$$
\begin{aligned}
& \sup _{0 \leq \mathfrak{t} \leq \omega} E\left|x^{(\eta+1)}(\mathfrak{t})-x^{(\eta)}(\mathfrak{t})\right|^{2} \leq M_{0} \prod_{i=1}^{\eta-1} \frac{\Gamma(i(2 a-1))}{\Gamma(i(2 a-1)+a)} \\
& {\left[3 L^{2} \Gamma(a) \omega^{a}(1+\omega)\left(1+\omega^{1-a}\right)\right]^{\eta},}
\end{aligned}
$$

provided that

$$
M_{0}:=\frac{\left.\left.3 K^{2}\left(1+E \mid w_{0}\right)\right|^{2}\right)(1+\omega)}{\Gamma(a)}\left(\frac{\omega}{2}+\frac{\omega^{a}}{a+1}\right) .
$$

Thus, for any $\phi, \theta \in N$ such that $\phi>\theta>0$,

$$
\begin{aligned}
\left\|x^{(\phi)}(\mathfrak{t})-x^{(\theta)}(\mathfrak{t})\right\|_{L^{2}(\mathbb{P})}^{2} & \leq \sum_{\eta=\theta}^{\phi}\left\|x^{(\eta+1)}(\mathfrak{t})-x^{(\eta)}(\mathfrak{t})\right\|_{L^{2}(\mathbb{P})}^{2} \\
& =\sum_{\eta=\theta}^{\phi} \int_{0}^{\omega} E\left|x^{(\eta+1)}(\mathfrak{t})-x^{(\eta)}(\mathfrak{t})\right|^{2} d \mathfrak{t} \\
\leq & M_{1} \sum_{\eta=\theta}^{\phi}\left[3 L^{2} \Gamma(a) \omega^{a}(1+\omega)\left(1+\omega^{1-a}\right)\right]^{\eta} \\
& \times \prod_{i=1}^{\eta-1} \frac{\Gamma(i(2 a-1))}{\Gamma(i(2 a-1)+a)} \rightarrow 0,
\end{aligned}
$$

for sufficiently large $\phi, \theta$ such that:

$$
M_{1}:=\frac{\left.3 K^{2}\left(1+E \mid w_{0}\right)\right|^{2}(1+\omega)}{\Gamma(a)}\left(\frac{\omega^{2}}{2(2+a)}+\frac{\omega^{a+1}}{(a+1)(2 a+1)}\right) .
$$


From Doob's maximal inequality for martingales,

$$
\begin{aligned}
& \sum_{\eta=1}^{\infty} \mathbb{P}\left[\sup _{0 \leq \mathfrak{t} \leq \omega}\left|x^{(\eta+1)}(\mathfrak{t})-x^{(\eta)}(\mathfrak{t})\right|>\frac{1}{\eta^{2}}\right] \\
& \leq M_{0} \sum_{\eta=1}^{\infty}\left[3 L^{2} \Gamma(a) \omega^{a}(1+\omega)\left(1+\omega^{1-a}\right)\right]^{\eta} \\
& \times \prod_{i=1}^{\eta-1} \frac{\Gamma(i(2 a-1))}{\Gamma(i(2 a-1)+a)} \eta^{4}<+\infty .
\end{aligned}
$$

The Borel cantelli lemma yields:

$$
\mathbb{P}\left\{\sup _{0 \leq \mathfrak{t} \leq \omega}\left|x^{(\eta+1)}(\mathfrak{t})-x^{(\eta)}(\mathfrak{t})\right|>\frac{1}{\eta^{2}} \text { for infinitely many } \eta\right\}=0,
$$

so there exist a random variable $x(\mathfrak{t})$ which is almost surely uniformly continuous on $[0, \omega]$, such that:

$$
x^{(\eta)}(\mathfrak{t})=x^{(0)}(\mathfrak{t})+\sum_{\theta=0}^{\eta-1}\left(x^{(\theta+1)}(\mathfrak{t})-x^{(\theta)}(\mathfrak{t})\right) \stackrel{\eta \rightarrow \infty}{\longrightarrow} x(\mathfrak{t}) .
$$

Since $x^{(\eta)}(\mathfrak{t})$ is t-continuous for any $\eta \in N$, so $x(\mathfrak{t})$ is also $\mathfrak{t}$-continuous. Therefore,

$$
\begin{aligned}
& w_{0}+\int_{0}^{\mathfrak{t}} b\left(p, x^{(\eta)}(p)\right) d p+a \int_{0}^{\mathfrak{t}}(\mathfrak{t}-p)^{a-1} \sigma_{1}\left(p, x^{(\eta)}(p)\right) d p \\
& +\int_{0}^{\mathfrak{t}} \sigma_{2}\left(p, x^{(\eta)}(p)\right) d B_{p} \stackrel{\eta \rightarrow \infty}{\longrightarrow} x(\mathfrak{t}),
\end{aligned}
$$

for a stochastic process $x(\mathfrak{t})$ satisfying (12).

Theorem 7. Under the conditions of Theorem 6, stochastic integral equation (12) has at most one solution.

Proof. Let $x_{1}(\mathfrak{t})$ and $x_{2}(\mathfrak{t})$ be solutions of stochastic integral equation (12), which have the initial conditions $x_{i}^{(0)}(\mathfrak{t})=\mathfrak{t}_{i}, 1 \leq i \leq 2$. Application of Cauchy-Schwartz inequality, the Itô Isometry, and Lipschitz condition, yield

$$
\begin{aligned}
E\left|x_{1}(\mathfrak{t})-x_{2}(\mathfrak{t})\right|^{2} \leq & 4 E\left|\mathfrak{t}_{1}-\mathfrak{t}_{2}\right|^{2}+4 L^{2}(1+\omega) \\
& \times \int_{0}^{\mathfrak{t}} E\left|x_{1}(p)-x_{2}(p)\right|^{2} d p \\
& +4 a L^{2} \omega^{a} \int_{0}^{\mathfrak{t}}(\mathfrak{t}-p)^{a-1} E\left|x_{1}(p)-x_{2}(p)\right|^{2} d p
\end{aligned}
$$

which can also be written as:

$$
E\left|x_{1}(\mathfrak{t})-x_{2}(\mathfrak{t})\right|^{2} \leq 4 E\left|\mathfrak{t}_{1}-\mathfrak{t}_{2}\right|^{2}+4 L^{2}(1+\omega) \omega^{1-a}
$$




$$
\begin{aligned}
& \times \int_{0}^{\mathfrak{t}} \mathfrak{t}^{a-1} p^{a-1}\left\{p^{1-a} E\left|x_{1}(p)-x_{2}(p)\right|^{2}\right\} d p \\
& +4 a L^{2} \omega^{a} \int_{0}^{\mathfrak{t}}(\mathfrak{t}-p)^{a-1} p^{a-1} \\
& \times\left\{p^{1-a} E\left|x_{1}(p)-x_{2}(p)\right|^{2}\right\} d p .
\end{aligned}
$$

Application of Corollary 4 yields:

$$
\begin{aligned}
E\left|x_{1}(\mathfrak{t})-x_{2}(\mathfrak{t})\right|^{2} \leq & 4 E\left|\mathfrak{t}_{1}-\mathfrak{t}_{2}\right|^{2} F_{2 a-1, a-1,2 a-1}\left(4 L^{2} \Gamma(a)\right. \\
& \left.\times\left\{(1+\omega) \omega^{1-a}+a \omega^{a}\right\} \mathfrak{t}^{2 a-1}\right) .
\end{aligned}
$$

Since, $x_{1}(\mathfrak{t})$ and $x_{2}(\mathfrak{t})$ are solutions of stochstic integral equation (12), with the initial conditions $x_{i}^{(0)}(\mathfrak{t})=\mathfrak{t}_{i}, 1 \leq i \leq 2$ therefore $\mathfrak{t}_{1}=\mathfrak{t}_{2}$ and hence

$$
E\left|x_{1}(\mathfrak{t})-x_{2}(\mathfrak{t})\right|^{2}=0 \text { for all } \mathfrak{t}>0,
$$

which proves the uniqueness.

\section{REFERENCES}

[1] M. Ahmed Boudref, A. Berboucha, New inequalities of Gronwall type for the stochastic differential equations, Random. Oper. Stoch. Equ., 23 (3) (2015), 151-159.

[2] Z. Denton, A. S. Vatsala, Fractional integral inequalities and applications, Comput. Math. Appl., 59 (3) (2010), 1087-1094.

[3] M. Hammi, M. Ali Hammi, Gronwall-Bellmann type integral inequalities and applications to global uniform asymptotic stability, CUBO A Math. J., 17 (3) (2015), 53-70.

[4] Q.-X Kong, X.-li Ding, A new fractional integral inequality with singularity and its application, Abst. Appl. Anal., 2012 (2012), Article ID 937908, 1-12.

[5] S.-Y Lin, Generalized Gronwall inequalities and their applications to fractional differential equations, J. Inequal. Appl., 549 (2013), 1-9.

[6] S.-Y Lin, New results for generalized Gronwall inequalities and their applications, Abst. Appl. Anal., 2014 (2014), Article ID 168594, 1-8.

[7] J. Shao, F. Meng, Gronwall-Bellmann type inequalities and their applications to fractional differential equations, Abst. Appl. Anal., 2013 (2013), Article ID 217641, 1-7.

[8] Q. Wu, A new type of the Gronwall-Bellmann inequality and its application to fractional stochastic differential equations, Cogent Math., 4 (1) (2017), 1-13. 
[9] H. Ye, J. Gao, Y. Ding, A generalized gronwall inequality and its application to a fractional differential equation, J. Math. Anal. Appl., 328 (2) (2007), 1075-1081.

[10] Z. Zhang, Z. Wei, A generalized Gronwall inequality and its application to fractional neutral evolution inclusions, J. Inequal. Appl., 45 (2016), 1-18.

[11] B. Zheng, Q. Fenq, New Gronwall-Bellmann type inequalities and applications in the analysis for solutions to fractional differential equations, Abst. Appl. Anal., 2013 (2013), Article ID 705126, 1-13.

[12] B. Zheng, Eplicit bounds derived by some new inequalities and applications in fractional integral equations, J. Inequal. Appl., 4 (2014), 1-12. 
